\title{
Gunshot Injury Causing Vascular, Bone and Genital Damages
}

\author{
Youness Jabbour ${ }^{1,2 *}$, Amine Slaoui ${ }^{1,2}$, Tarik Karmouni ${ }^{1,2}$, Khalid Elkhader ${ }^{1,2}$, Abellatif Koutani ${ }^{1,2}$ and Ahmed Iben Attya Andaloussi ${ }^{1,2}$ \\ ${ }^{1}$ Urologie B Department, Ibn Sina Teaching Hospital Rabat, Morocco \\ ${ }^{2}$ Faculty of Medicine and Pharmacy of Rabat, Morocco
}
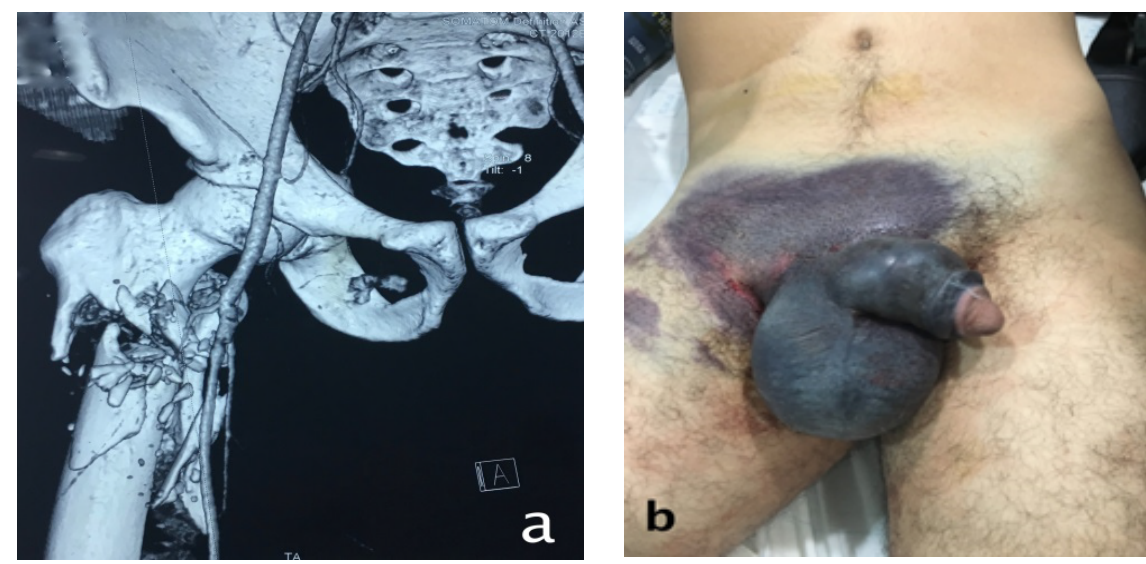

Figure 1: Computed tomography scan revealed a complex sub-trochanteric fracture of the femur with a wound at the superficial femoral artery.

Figure 2: Ecchymosis of external genitalia with deviation and swelling of penile.

\section{Clinical-Medical Image}

We report a case of a young patient brought to emergency department by police ambulance for management of gunshot injury after getting shot by a policeman. First clinical examination found a conscient and hemodynamically stable patient and a huge ecchymosis of external genitalia with deviation and swelling of penile with a puncture wound on the left side of the base of the penis probably corresponding to the exit orifice of the bullet. Ultrasonography with Doppler was found a bilateral rupture of cavernous bodies as well as a left testicular hematoma without rupture of the tunica albuginea and an extensive hematoma of the right inguinal region. CT-scan revealed a complex sub-trochanteric fracture of the femur with a wound at the superficial femoral artery (Figure 1). Surgical repair was performed with initially a venous bypass for the femoral artery and suture of the cavernous bodies. No urethral lesion was found and he was operated one month later for his fractured femur (Figure 2).

External genital gunshot injuries are rare both in war field and in civil practice. Its occurrence in civilian practice makes it possible to establish good diagnostic and therapeutic strategies given the availability of complementary exploration means.

Ballistic traumas are the result of penetration into the body of a projectile. No physical theory can predict with certainty the behavior of a projectile in the human body. Damages are greater when projectiles collide with dense structures such as bones resulting in a high energy transfer.

CT-scan help to assess with great sensitivity the associated lesions in this case it allowed diagnosing other serious and life-threatening associated injuries.

Keywords: Penile fracture; Gunshot; Computed tomography

*Corresponding author: Youness Jabbour, Resident at Urology B Department, Ibn Sina Teaching Hospital, Rabat, Morocco, Tel No: +212-660278360, , E-mail: uro.jabbour@gmail.com

Citation: Jabbour Y, Slaoui A, Karmouni T, Elkhader K, Koutani A, Andaloussi AIA (2018) Gunshot Injury Causing Vascular, Bone and Genital Damages . Int J Clin Med Imaging 5: 616. doi:10.4172/2376-0249.1000616

Copyright: (C) 2018 Jabbour Y, et al. This is an open-access article distributed under the terms of the Creative Commons Attribution License, which permits unrestricted use. distribution. and reproduction in any medium. provided the original author and source are credited. 